\title{
Realidad de la derivación a otorrinolaringología desde la atención primaria
}

\section{Reality of the derivation to otorhinolaryngology from primary attention}

\author{
Carolina Der $\mathrm{M}^{1}$, María Paz Salin $\mathrm{V}^{2}$, Magaly Iñiguez $\mathrm{C}^{3}$, Pilar Valenzuela $\mathrm{M}^{3}$.
}

\begin{abstract}
RESUMEN
La dificultad de acceso a la atención de especialista en el sistema público es un problema serio para sus usuarios. Los criterios utilizados para priorizar la atención, son el diagnóstico y los síntomas enunciados en la interconsulta (IC) por el profesional que deriva Los objetivos de este trabajo son dar a conocer la realidad del Hospital Dr. Sótero del Río, identificar causas administrativas y médicas de derivación inadecuada, e intentar sentar las bases para dar solución a estos problemas. De un total de 7.750 interconsultas en lista de espera de atención por otorrinolaringología, hasta junio de 2003, se extrajo una muestra de 1.150 interconsultas y se realizó una base de datos con la información aportada Un subgrupo de 115 pacientes, fueron evaluados por otorrinolaringólogos del servicio. Resultados: 1 96\% de las interconsultas estaban incompletas, hubo un 5\% de error en derivación, el 74\% de las IC no tenían fundamento diagnóstico, la coincidencia diagnóstica alcanzó el 25\%. B cuadro clínico descrito en la interconsulta, muchas veces no fue un dato confiable para dar prioridad en la atención de los pacientes. Las soluciones al problema de atención por especialista dependen de un trabajo cooperativo entre docentes universitarios, otorrinolaringólogos, médicos generales y personal administrativo.
\end{abstract}

Palabras clave: Listas de espera, derivación desde atención primaria.

\section{SUMMARY}

The difficulty in gaining access to a specialist in the public system is a severe problem for the users. The criteria applied to priorize attention are diagnosis and the symptoms enounced in the interconsultation (IC) by the derivating professional. The objectives of this paper are to inform about the reality of Dr. Sotero del Rio Hospital, to identify administrative and medical causes for inadequate derivation, and to try to set the bases to solve these problems. Of a total of 7.750 interconsultations on a waiting list for otolaryngology attention, up to June 2003, a sample of 1.150 interconsultations was extracted and a data base was developed with the information obtained. A sub-group of 115 patients were evaluated by otorhinolaryngologists from the service. Results: 96\%

\footnotetext{
${ }^{1}$ Médico Hospital Dr. Sótero del Río.

${ }^{2}$ Médico Hospital del Salvador.

${ }^{3}$ Médico Hospital Cínico Pontificia Universidad Católica de Chile.
} 
of the interconsultations were incomplete, there was a 5\% error in derivation, $74 \%$ of the IC did not have a diagnostic basis, diagnostic coincidence amounts to $25 \%$. The clinical picture described in the interconsultation was many times not a reliable reference to give priority to the attention of patients. Solutions to the problem of attention by a specialist depend on a cooperative work between university teachers, otorhinolaryngologists, general practitioners and administrative staff.

Key words: Wait lists, derivation from primary attention.

\section{INTRODUCCIÓN}

En nuestro país, la salud se caracteriza por ser un sistema mixto de atención, en el que existe un sector público y uno privado. Estos dos sistemas actúan como vasos comunicantes, disminuyendo el número de pacientes de uno en función del aumento del otro. La realidad social y económica de cada momento histórico definirá cuál de los dos abarcará un mayor volumen de pacientes.

En la actualidad el sistema público de salud chileno está organizado en tres niveles de atención; primaria, que abarca Consultorios y Servicios de Atención Primaria de Urgencia (SAPU), secundaria, constituida por hospitales con especialidades básicas y, terciaria, con hospitales de alta complejidad. $\mathrm{日}$ sistema público es el que atiende aproximadamente al $80 \%$ de la población chilena, por lo tanto, la dificultad de acceso a la atención médica en los últimos años es un problema grave que perjudica enormemente a sus usuarios. Este tema se enfoca mayoritariamente a las listas de espera quirúrgicas, pues dan una impresión más dramática a ojos de la opinión pública, pero no podemos dejar de recalcar que existen enormes listas de espera de atención médica (de policlínico), generadas a partir de interconsultas cursadas por otros profesionales de la salud.

La otorrinolaringología es una especialidad que se caracteriza por tener la mayoría de sus consultas catalogadas con el carácter de "no urgente", por esto las autoridades de salud prefieren aplazar la resolución de los problemas de atención que se detectan, para resolver temas más urgentes. Precisamente por esto, es nuestro deber como especialistas el dar a conocer al resto de la comunidad la realidad de atención en nuestros centros de trabajo.

Los autores del presente trabajo de investigación están vinculados al Hospital Dr. Sótero del Río, nivel de atención terciario de salud del área sur oriente de Santiago, con una población asignada de 1.347.210 habitantes, procedentes de las comunas de Puente Alto, La Forida, San José de Maipo, Pirque, San Ramón, La Granja y La Pintana'. Los entes derivadores de pacientes al hospital son 30 consultorios con sus respectivos Servicios de Atención Primaria de Urgencia (SAPU), 2 hospitales tipo B, el Centro de Diagnóstico y Tratamiento (CDT) y el Servicio de Urgencia, estos dos últimos con sus dependencias adosadas al Hospital Dr. Sótero del Río.

Desde el punto de vista de lo que las autoridades denominan "gestión hospitalaria", el año 2002 este Hospital se hizo merecedor del premio a la excelencia, otorgado a todos sus servicios, incluido el servicio de otorrinolaringología. Este servicio contaba en ese año con once especialistas, correspondiente a un total de 253 horas médicas de atención semanales. Estos profesionales (utilizando los boxes de atención mañana y tarde) lograban una atención de 16.000 pacientes por año, de los cuales un tercio correspondían a casos nuevos. Para establecer prioridad en la atención y así elegir los casos de mayor gravedad o urgencia, se usaban como criterios de selección el diagnóstico y los síntomas enunciados en la interconsulta escrita por el profesional que derivaba a la especialidad.

En principio, esto parece adecuado, sin embargo, en junio de 2003, en el Servicio de Salud Metropolita no Sur-Oriente (SSMSO) existíaunalista de espera de 32.125 interconsultas totales, de las cuales 7.550 correspondían a otorrinolaringología, conformando la 
segunda lista de espera más larga, luego de oftalmología?. Debido a esta situación, nos propusimos contribuir a la identificación de los problemas que generan y perpetúan la lista de espera del policlínico de otorrinolaringología, junto con proponer ideas que aporten a la solución de este problema, desde la perspectiva del médico especialista

\section{OBJETIVOS}

1. Identificar causas administrativas de derivación inadecuada

a) Describir las características del grupo de pacientes derivados, origen y distribución mensual de las interconsultas.

b) Evaluar la calidad del registro de los datos de identificación de los pacientes derivados.

c) Evaluar el manejo de las interconsultas por el Servicio de Orientación Médico Especialista (SOME).

2. Identificar causas médicas de derivación inadecuada

a) Cuantificar los diagnósticos que generan la derivación a otorrinolaringología e identificar al profesional que deriva y su especialidad.

b) Verificar la congruencia entre el diagnóstico enunciado, los síntomas descritos y la conclusión diagnóstica del otorrinolaringólogo luego de la primera consulta.

3. Proponer ideas para la solución de los problemas desde la perspectiva del médico especialista.

\section{MATERIAL Y MÉTODO}

Se realizó un estudio transversal y descriptivo, entre abril y junio de 2003. 日 grupo a estudiar estuvo compuesto por interconsultas de pacientes derivados al Servicio de Otorrinolaringología del Hospital Dr. Sótero del Río, quese encontraban en lalista de espera registrada a junio 2003 (7.750 Interconsultas). De estas interconsultas se tomó una muestra aleatoria de 1.150 interconsultas correspondientes al $15 \%$ del total.

En una base de datos Excel se registraron los antecedentes generales del paciente descritos en la interconsulta (nombre, edad, sexo, dirección, telé fono, № de RUT, № de ficha), tipo e identificación del profesional que deriva y el origen de la interconsulta. Se consignó el diagnóstico enunciado por el profesional derivador y la hipótesis diagnóstica planteada por el otorrinolaringólogo a partir de la clínica descrita, estos dos diagnósticos se compararon buscando coincidencia.

Se seleccionó al azar un subgrupo de 115 pacientes (correspondientes al 10\% de la muestra) que fueron examinados por otorrinolaringólogos del servicio, quienes luego de la primera consulta plantearon un diagnóstico, el cual se comparó con la información entregada en la interconsulta.

日 análisis estadístico de los datos se llevó a cabo utilizando test de proporciones, evaluando los intervalos de confianza.

\section{RESULTADOS}

Del total de las 1.150 interconsultas analizadas, un $55 \%$ corresponden a pacientes de sexo femenino y $45 \%$ de sexo masculino, diferencia estadísticamente significativa (mediante test de proporciones, IC 95,0\%). 1 grupo etáreo más prevalente son los niños de 0 a 9 años (29\% de las interconsultas), seguido por los adultos mayores de 60 años (21\% de interconsultas) (Tabla 1).

Tabla 1. Distribución por edad y sexo de 1.150 pacientes

\begin{tabular}{|lcccccccccc|}
\hline Sexo & $\begin{array}{c}0-4 \\
\text { Años }\end{array}$ & $\begin{array}{c}5-9 \\
\text { años }\end{array}$ & $\begin{array}{c}10-19 \\
\text { años }\end{array}$ & $\begin{array}{c}20-29 \\
\text { años }\end{array}$ & $\begin{array}{c}30-39 \\
\text { años }\end{array}$ & $\begin{array}{c}40-49 \\
\text { años }\end{array}$ & $\begin{array}{c}50-59 \\
\text { años }\end{array}$ & $\begin{array}{c}60-69 \\
\text { años }\end{array}$ & $\begin{array}{c}70 \text { y } \\
\text { más }\end{array}$ & Total \\
\hline Femenino & 69 & 79 & 87 & 46 & 72 & 55 & 79 & 62 & 85 & 634 \\
Masculino & 89 & 94 & 102 & 27 & 34 & 20 & 52 & 40 & 58 & 516 \\
Total & 158 & 173 & 189 & 73 & 106 & 75 & 131 & 102 & 143 & 1.150 \\
\hline
\end{tabular}


La Figura 1, muestra la distribución geográfica de procedencia de las interconsultas en espera, siendo los centros de la comuna de Puente Alto los que más derivan (49\%), cuya proporción en comparación al resto de los centros derivadores fue estadísticamente significativa. Encontramos un 3\% de interconsultas correspondientes a comunas no beneficiarias del hospital. Llama la atención la disparidad en cuanto al número de pacientes derivados de las distintas comunas, independiente del número de habitantes de éstas, especialmente en el caso de La Horida, que siendo una de las más populosas de la zona presenta un muy bajo porcentaje de pacientes esperando evaluación. Al investigar al respecto, nos informamos que el Centro de Referencia de Salud (CRS) de La Horida cuenta con cupos de derivación directos al especialista del nivel terciario de atención (sin pasar por el SOME del Hospital), siendo los Médicos Generales Familiares los que deciden la derivación inmediata según su impresión diagnóstica. Revisamos nuestros registros de servicio y encontramos que todos los pacientes de La Horida atendidos recientemente correspondían a interconsultas cursadas a fines de 2002 y 2003. Este hecho es muy beneficioso para los habitantes de La Horida, pero debemos recordar que hay pacientes de las otras comunas (específicamente Puente Alto) esperando ser atendidos desde 1999.

日 tiempo de espera promedio de los pacientes fue de 35,3 meses y el promedio mensual de nuevas interconsultas recibidas cada mes fue de 180. La Figura 2 resume la estacionalidad de la derivación y se puede observar que el $71 \%$ se concentra entre el segundo y el tercer trimestre del año.

\section{Registro de los datos de identificación de los pacientes derivados}

En cuanto al manejo administrativo de las interconsultas desde los centros derivadores, destaca el hecho que el registro completo de los datos se observó sólo en el 4\% de las interconsultas evaluadas. Esto es, en el $96 \%$ de las interconsultas evaluadas, no se escribió toda la información solicitada referente al paciente. Los datos más prevalentes fueron primer nombre y primer apellido que se encontraron en el $100 \%$ de las interconsultas, seguido del № de RUT con un $83 \%$,

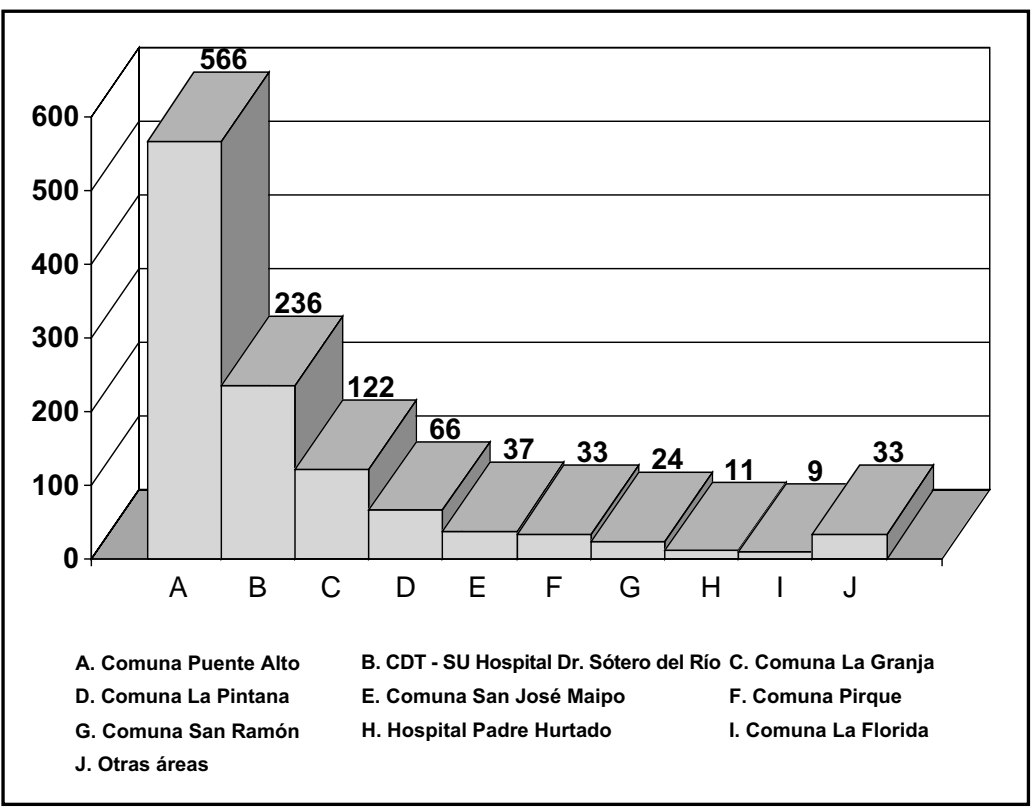

Figura 1. Distribución geográfica del origen de interconsultas. 


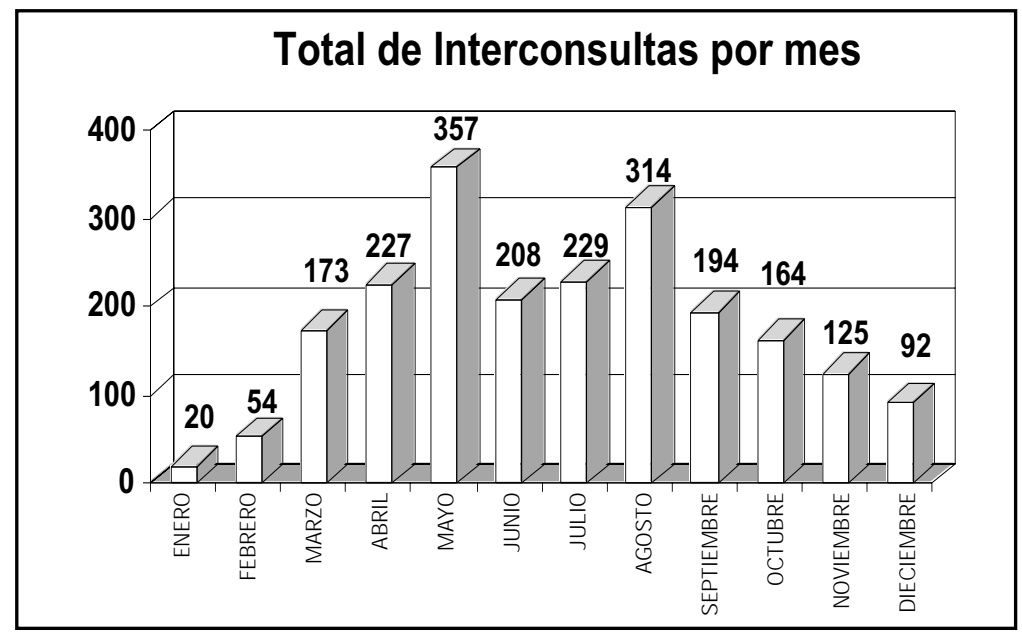

Figura 2. Distribución estacional de las interconsultas.

teléfono con un $79 \%$, fecha de nacimiento con un $77 \%$, dirección con un $68 \%$ y № Ficha con un $42 \%$. Esta información incompleta dificulta el contactar a los pacientes para coordinar la asistencia a la consulta, cuando les corresponda.

Si observamos el tiempo que transcurre desde la emisión de la interconsulta hasta su recepción en el SOME, vemos que el promedio es de ocho días con un rango de 0 a 727 días. Por otro lado, hay un $8 \%$ de interconsultas que no tienen timbre de recepción, pero que se encontraban en el SOME al momento del estudio, no sabemos si éstas ingresaron por una vía paralela al sistema o por error no fueron recibidas en forma correcta. Llama la atención que al momento del estudio no existía registro de las interconsultas recibidas y éstas se almacenaban en cajas cuyo único tipo de clasificación era el año y el servicio al cual iban dirigidas. Un 3\% de las interconsultas provenían de comunas que no forman parte del SSMSO y detectamos errores en un $2 \%$ de las interconsultas recibidas por el SOME: un $1 \%$ estaban repetidas, es decir había una duplicidad de derivación para un mismo paciente, y un $1 \%$ eran interconsultas dirigidas a otros servicios cuyo nombre comenzaba con la letra "O” como oftalmología u oncología.

\section{Diagnósticos que generan la derivación a otorrinolaringología, profesional que deriva y su especialidad}

Para los 1.150 pacientes de la muestra, se registraron un total de 1.358 diagnósticos con un promedio de tres diagnósticos por paciente y con un rango de 0 a 4. Encontramos 77 tipos de diagnósticos otorrinolaringológicos propuestos, los cuales para facilitar su análisis fueron organizados en los 10 grupos que se muestran en la Figura 3, siendo estadísticamente significativala mayor frecuencia de hipoacusia (32,2\%) y patología adenoamigdalina (32\%), respecto al resto de las patologías. Cabe destacar que el $4,3 \%$ de la muestra estaba rotulada como "Urgente", sin embargo, al analizar los datos descritos en la interconsulta en cuanto a síntomas o diagnóstico, sólo un tercio de éstos podrían corresponder a una real urgencia.

日 $67 \%$ de las interconsultas analizadas no tenían identificación del profesional que las enviaba y específicamente no indicaba su acreditación académica

日 $15 \%$ de las interconsultas fueron enviadas por médicos especialistas, siendo las especialidades que más derivaron en orden decreciente: neurología, oftalmología, pediatría y broncopulmonar. 


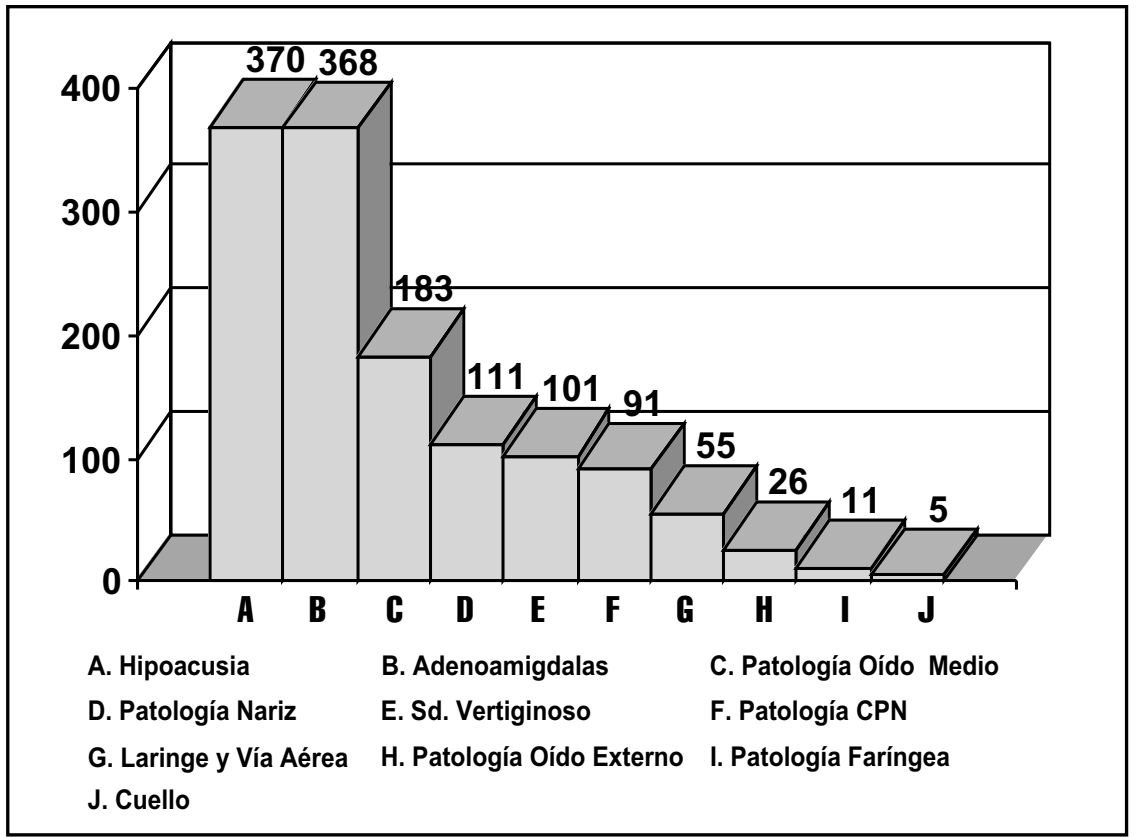

Figura 3. Distribución de interconsultas según grupo diagnóstico.

日 $10 \%$ de las interconsultas fueron enviadas por médicos generales y el $5 \%$ por médicos del Servicio de Urgencia.

日 3\% de las interconsultas fueron emitidas por otros profesionales de la salud, siendo los que más derivaban en orden decreciente: enfermeras, odontólogos y fonoaudiólogos.

Sólo el 39\% de los profesionales se identificó con nombre o RUT. Esto tiene implicancia tanto en lo que respecta a asumir responsabilidad sobre un acto médico como en la comunicación expedita entre el médico tratante y el especialista evaluador.

Congruencia entre el diagnóstico enunciado por el médico que deriva y la hipótesis diagnóstica del otorrinolaringólogo basado en la descripción del cuadro clínico en la interconsulta

Para realizar este análisis, 2 médicos otorrinolaringólogos, en conjunto determinaron si el cuadro clínico descrito se correlacionaba con el diagnóstico propuesto en la interconsulta por el profesional derivador. De las 1.150 interconsultas revisadas, en 415 de ellas (36\%) sólo se enunciaba el diagnóstico, sin describir signos o síntomas, lo que no permitió realizar el análisis de congruencia. De las 735 restantes, sólo hubo congruencia en 311 (27\% del total de la muestra), lo cual equivale a menos de la mitad de aquellas que consignan descripción clínica.

Se seleccionaron al azar 115 interconsultas, estos pacientes fueron atendidos por los médicos del servicio en el contexto de su consulta habitual. Se encontró congruencia entre el diagnóstico otorrinolaringológico y el diagnóstico propuesto por del profesional derivador o el cuadro clínico descrito por éste, sólo en el $25 \%$ de los casos. Al buscar coincidencia entre el diagnóstico otorrinolaringológico, el propuesto por el médico derivador y el cuadro clínico descrito, ésta se encontró sólo en el $10 \%$ de los casos. Cabe destacar que $9 \%$ de estos 115 pacientes no tenían diagnóstico de re sorte otorrinolaringológico y debieron haber sido derivados inicialmente a otras especialidades. 


\section{DISCUSIÓN}

En la actualidad existe una gran dificultad de acceso a la evaluación por especialidades médicas de la atención terciaria, esto se refleja claramente en la enorme lista de espera de atención de otorrinolaringología que llega a 7.550 interconsultas. Al hacer el análisis de la muestra de 1.150 interconsultas pudimos dilucidar numerosos problemas que se resumen a continuación:

\section{Problemas administrativos}

1. Identificación incompleta de los pacientes (96\% de Interconsultas), tema que compete a los lugares derivadores y su personal.

2. Existen diferencias importantes en cuanto a prioridad de acuerdo a la comuna de origen (la comuna de La Horida tiene cupos directos a la atención por la especialidad mientras que la comuna de Puente Alto tiene una gran lista de espera con pacientes en espera desde 1999).

3. No existe registro del SOME, llevando a recepción de interconsultas duplicadas, de otras áreas $u$ otras especialidades.

4. Hay $5 \%$ de interconsultas equivocadas por problemas administrativos previamente identificados.

\section{Problemas médicos}

1. Falta de identificación de los profesionales que derivan.

2. Uso inadecuado del rótulo "Urgente".

3. Fundamentos diagnósticos sólo en el $74 \%$ de las interconsultas. Estos fundamentos son congruentes con el diagnóstico enunciado, entre un 9 y un $39 \%$ de los casos, según patología.

4. Existe concordancia clínica o diagnóstica entre el profesional que deriva y el otorrinolaringólogo sólo en el $25 \%$ de los casos.

5. Hay un $9 \%$ de pacientes que por el diagnóstico enunciado no corresponden a la especialidad.

Dada la gran cantidad de problemas encontrados quisimos dar a conocer algunas Propuestas de Solución.

\section{Soluciones a corto plazo}

Los problemas administrativos se podrían resolver prácticamente en su totalidad, si existiera una base de datos computarizada con campos obligatorios para registrar las interconsultas recibidas en el SOME Así, al momento de ingresar los datos del paciente, el sistema detectaría los casos duplicados y correspondientes a otras comunas.

Es responsabilidad de los profesionales de la salud, que derivan a la especialidad, enviar las interconsultas completas con todos los datos del paciente.

Creemos que, en función del principio de justicia, la prioridad de atención debe otorgarse por gravedad del cuadro o por fecha de emisión y no por comuna de origen.

\section{Soluciones a mediano plazo}

Nuestra capacidad actual de responder a la demanda de atención, considerando que tenemos 253 horas médicas contratadas en el servicio, nos permite generar 412 cupos para pacientes nuevos al mes $^{3}$. B servicio de urgencia envía un promedio de 240 pacientes mensuales que requieren de atención inmediata (el mismo día en que se solicita la evaluación), lo que deja 172 cupos para interconsultas nuevas de los consultorios. Recordemos que el promedio mensual de interconsultas recibidas es de 180, por lo tanto una vez resueltas todas las atenciones pendientes, no debería generarse una nueva lista de espera.

Sin embargo, para resolver la lista de espera existente, sería necesario que cada médico especialista de nuestro servicio atendiera dos policlínicos semanales de tres horas y media cada uno, atendiendo a cuatro pacientes por hora, en un horario extraordinario.

\section{Soluciones a largo plazo}

日 alto porcentaje de diagnósticos erróneos enunciados por los profesionales que derivan, nos obliga a realizar una evaluación de la docencia médica de pregrado. Existen errores conceptuales 
graves, que evidencian una importante falta de conocimiento. A modo de ejemplo citamos los casos en los cuales el profesional deriva al paciente con el diagnóstico de hiperplasia adenoídea y a continuación describe las características de una dislalia La formación otorrinolaringológica del mé dico general está en manos de nosotros los especialistas y es posible que en el afán de compartir con los alumnos todos nuestros conocimientos, más que destacar lo que les será de utilidad en su práctica diaria, les estemos entregando un exceso de información, la cual no tendrá para ellos utilidad clínica en el futuro. Es por esto que cremos que, al menos en parte, la solución a largo plazo gira en torno a orientar los cursos de pregrado a la práctica del médico general y no a la formación de un "pequeño especialista".

\section{CONCLUSIONES}

日 diagnóstico o cuadro clínico descrito en la interconsulta de derivación a otorrinolaringología no es un dato confiable para otorgar prioridad a la atención de los pacientes. Dada la baja concordancia entre la información entregada en las interconsultas por el médico derivador y aquella objetivada después por el especialista, éste no debe asumir la responsabilidad médico legal que implica priorizar pacientes a partir de esta información, pues desde el momento en que así lo determina, realiza un acto médico con todo lo que ello implica.

Las soluciones al problema de nuestra lista de espera de policlínico y el evitar que estos problemas se perpetúen en el futuro, requieren de un trabajo cooperativo entre docentes universitarios, otorrinolaringólogos, médicos generales y personal administrativo.

\section{BIBLIOGRAFÍA}

1. Estadísticas SMSSO. Indicadores Biodemográficos. Página web del Servicio de Salud Metropolitano Sur-Oriente www.ssmso.cl. 2003.

2. Chile Estadístico. Demografía y Vitales 20002005. Página web Instituto Nacional de Estadísticas, www.ine.cl

3. Listas de espera para atención por especialidades médicas en el SSMSO. 2003.

4. Archivo de programación médica del Centro de Diagnóstico Terapéutico. 2003. 